\title{
The cutting power of preparation
}

\author{
Olivier Tercieux • Mark Voorneveld
}

Received: 11 January 2008 / Accepted: 29 January 2009 / Published online: 18 February 2009 (C) The Author(s) 2009. This article is published with open access at Springerlink.com

\begin{abstract}
In a strategic game, a curb set (Basu and Weibull, Econ Lett 36:141-146, 1991 ) is a product set of pure strategies containing all best responses to every possible belief restricted to this set. Prep sets (Voorneveld, Games Econ Behav 48:403-414, 2004) relax this condition by only requiring the presence of at least one best response to such a belief. The purpose of this paper is to provide sufficient conditions under which minimal prep sets give sharp predictions. These conditions are satisfied in many economically relevant classes of games, including supermodular games, potential games, and congestion games with player-specific payoffs. In these classes, minimal curb sets generically have a large cutting power as well, although it is shown that there are relevant subclasses of coordination games and congestion games where minimal curb sets have no cutting power at all and simply consist of the entire strategy space.
\end{abstract}

Keywords Curb sets - Prep sets - Supermodular games · Potential games · Congestion games

\section{Introduction}

Set-valued coarsenings of the Nash equilibrium concept have proven to possess a number of appealing properties. For instance, set-valued solutions adequately model

O. Tercieux

Paris-Jourdan Sciences Economiques (PSE) and CNRS, Paris, France

M. Voorneveld

Department of Econometrics and Operations Research, Tilburg University, Tilburg, The Netherlands

M. Voorneveld (凶)

Department of Economics, Stockholm School of Economics, Box 6501, 11383 Stockholm, Sweden e-mail: Mark.Voorneveld@hhs.se 
the intuition that people live by rules and principles (rules of thumb, the Ten Commandments, teetotalism, etc.) that restrict behavior without determining it uniquely, and they provide a characterization of the limit behavior of many plausible models of strategy adjustment, cf. Hurkens (1995); Young (1998); Kosfeld et al. (2002); Tercieux (2006), and Kets and Voorneveld (2008).

Set-valued solutions, however, may provide very unsharp predictions: non-equilibrium strategies may be included and in some games the solution may have no cutting power whatsoever and simply consist of the entire strategy space. Hence, to evaluate the practical appeal of such concepts, it is important to provide economically relevant classes of games where they have considerable cutting power.

The current paper focusses on the cutting power of minimal prep sets ('prep' is short for 'preparation'). This set-valued solution concept, introduced in Voorneveld (2004, 2005), combines a standard rationality condition, stating that the set of recommended strategies to each player must contain at least one best reply to whatever belief he may have that is consistent with the recommendations to the other players, with players' aim at simplicity, which encourages them to select a set of strategies that is as small as possible. Think of the set of recommendations to a player in a minimal prep set as a well-packed suitcase for a holiday: you want to be prepared for different kinds of weather, but bringing all five of your umbrellas and all seven bathing suits may be overdoing it. This distinguishes minimal prep sets from $(a)$ minimal curb sets (Basu and Weibull 1991), which are product sets of pure strategies containing not just some, but all best responses to beliefs restricted to the recommendations to the remaining players, and (b) persistent retracts (Kalai and Samet 1984), which also require the recommendations to each player to contain at least one best reply to beliefs in a small neighborhood of the beliefs restricted to the recommendations to the other players. Voorneveld $(2004,2005)$ gives a general existence proof and provides relations with Nash equilibria, rationalizability, minimal curb sets, and persistent retracts. Voorneveld etal. (2005) provide axiomatizations of minimal prep sets and minimal curb sets. Kets and Voorneveld (2008) show that appealing models of strategic adjustment eventually settle down in minimal prep sets.

The current paper focuses on a number of economically relevant classes of games with pure Nash equilibria (As a complement, we are investigating the cutting power of minimal prep sets in games without such equilibria in ongoing research). Indeed, pure Nash equilibria are appealing due to their simplicity, whereas the use of mixed strategies is sometimes (think of one-shot games) subject to criticism; see Luce and Raiffa (1957, Sect. 4.10) and Osborne and Rubinstein (1994, Sect. 3.2) for a critical appraisal of the use of mixed strategies.

What can one say about the cutting power of minimal prep sets in games with pure Nash equilibria? By definition, each pure Nash equilibrium corresponds with a singleton minimal prep set, but it is easy to construct games where minimal prep sets-in addition to the game's pure Nash equilibria_-select other sets as well. See Fig. 4 for an example. Therefore, it is of interest to see $(a)$ whether in economically appealing classes of games with pure Nash equilibria, the cutting power of minimal prep sets is as sharp as possible, in the sense that it picks out only the pure Nash equilibria, and $(b)$ to compare this cutting power with that of its nearest sibling, minimal curb sets. 
The main results of this paper are the following. Proposition 3.1 provides sufficient conditions under which the collection of minimal prep sets coincides with the collection of pure Nash equilibria. After a simple illustration of this result by means of a classical pure saddle-point theorem of Shapley (1964) for zero-sum games, it is shown to apply to two well-known classes of games which together cover a large range of economic applications.

The first class, studied in Sect. 4, consists of supermodular games, games where the best-response correspondences have certain monotonicity properties (Topkis 1978). Milgrom and Roberts (1990) and Topkis (1998) provide numerous applications, including search models, facility location, arms races, and oligopoly models.

The second class, studied in Sect. 5, consists of potential games, in particular the most general class of potential games of Monderer and Shapley (1996) and the bestresponse potential games of Voorneveld (2000). These potential games have applications to, for instance, congestion games (Rosenthal 1973), oligopoly models (Slade 1994), coalition formation (Slikker 2001), and the financing of public goods (Koster et al. 2003). Section 5.3 extends the analysis to the congestion games of Quint and Shubik (1994), which are typically not potential games.

When it comes to the cutting power of minimal curb sets in these classes of games, the results come in two variations. Firstly, in generic finite games belonging to these two classes of supermodular and potential games, the collections of minimal curb sets, minimal prep sets, pure Nash equilibria, and strict Nash equilibria all coincide; see the remarks following Propositions 4.1 and 5.1. ${ }^{1}$ This has an important consequence, since it is within minimal curb sets that many intuitive models of strategic adjustment settle down. Hence, in generic finite supermodular or potential games, these processes necessarily converge toward a strict Nash equilibrium.

Secondly, although these genericity results are of interest in their own right, many classes of games have additional structure, making them congeneric. To enhance this point, we also provide practically relevant subclasses of games where minimal prep sets give sharp predictions, whereas minimal curb sets have no cutting power whatsoever and simply consist of the entire strategy space: a class of coordination games in Sect. 4.3 and a class of minority games in Sect. 5.2. Minority games model situations where players strive to be in the most exclusive of two groups, for one of many possible reasons: standing out from the crowd might give status; one would prefer to choose the less crowded of two roads to work; if demand for a good is larger than supply, one would rather be a supplier, etc. See Moro (2003) for an introduction to minority games and Challet et al. (2004) for a book containing many of the path-breaking papers and applications to phenomena in financial markets.

The material is organized as follows. Section 2 contains preliminaries. In Sect. 3 we provide sufficient conditions for the collection of minimal prep sets and the collection of pure Nash equilibria to coincide and a give a simple illustration in the setting of zero-sum games. Applications to supermodular games are given in Sect. 4,

\footnotetext{
1 To avoid confusion: these genericity results do not follow from earlier ones in Voorneveld (2004, 2005): genericity is always defined with respect to a given domain and a property that is generic in one domain is not necessarily generic in another. For instance, the defining property 'being zero-sum' is clearly generic in zero-sum games, but is not a generic property in finite games.
} 
applications to potential games and the congestion games of Quint and Shubik (1994) in Sect. 5.

\section{Preliminaries}

A (strategic) game is a tuple $G=\left\langle N,\left(A_{i}\right)_{i \in N},\left(u_{i}\right)_{i \in N}\right\rangle$, where $N$ is a nonempty, finite set of players, each player $i \in N$ has a nonempty set of pure strategies (or actions) $A_{i}$ and a von Neumann-Mergansers utility function $u_{i}: \times_{j \in N} A_{j} \rightarrow \mathbb{R}$. A game is finite if each player has a finite set of pure strategies. Write $A=\times_{i \in N} A_{i}$ and for each $i \in N, A_{-i}=\times_{j \in N \backslash\{i\}} A_{j}$.

Payoffs are extended to mixed strategies in the usual way. Assuming each $A_{i}$ to be a topological space, $\Delta\left(A_{i}\right)$ denotes the set of Boreal probability measures over $A_{i}$. Using a common, minor abuse of notation, $\alpha_{-i}$ denotes both an element of $\times_{j \in N \backslash\{i\}} \Delta\left(A_{j}\right)$ specifying a profile of mixed strategies of the opponents of player $i \in N$, and the probability measure it induces over the set $A_{-i}$ of pure strategy profiles of his opponents. Beliefs of player $i$ take the form of such a mixed strategy profile. Similarly, if $B_{i} \subseteq A_{i}$ is a Boreal set, then $\Delta\left(B_{i}\right)$ denotes the set of Boreal probability measures with support in $B_{i}$ :

$$
\Delta\left(B_{i}\right)=\left\{\alpha_{i} \in \Delta\left(A_{i}\right) \mid \alpha_{i}\left(B_{i}\right)=1\right\}
$$

As usual, $\left(a_{i}, \alpha_{-i}\right)$ is the profile of strategies where player $i \in N$ plays $a_{i} \in A_{i}$ and his opponents play according to the mixed strategy profile $\alpha_{-i}=\left(\alpha_{j}\right)_{j \in N \backslash\{i\}} \in$ $\times_{j \in N \backslash\{i\}} \Delta\left(A_{j}\right)$.

Let $\Gamma$ denote the set of all games satisfying the following weak measurability assumption on the players' utility functions: for each player $i \in N$, for each $a_{i} \in A_{i}$ and each $\alpha_{-i} \in \times_{j \in N \backslash\{i\}} \Delta\left(A_{j}\right)$, the expected payoff $u_{i}\left(a_{i}, \alpha_{-i}\right)=\int_{A_{-i}} u_{i}\left(a_{i}, a_{-i}\right)$ $\mathrm{d} \alpha_{-i}$ is well-defined and finite. The set $\Gamma$ contains, in particular, all (mixed extensions of) finite strategic games.

Let $i \in N$ and let $\alpha_{-i} \in \times_{j \in N \backslash\{i\}} \Delta\left(A_{j}\right)$ be a belief of player $i$. The set

$$
B R_{i}\left(\alpha_{-i}\right)=\left\{a_{i} \in A_{i} \mid \forall b_{i} \in A_{i}: u_{i}\left(a_{i}, \alpha_{-i}\right) \geq u_{i}\left(b_{i}, \alpha_{-i}\right)\right\}
$$

is the set of pure best responses of player $i$ against $\alpha_{-i}$.

We recall the definitions of minimal curb sets and minimal prep sets. Let $G=$ $\left\langle N,\left(A_{i}\right)_{i \in N},\left(u_{i}\right)_{i \in N}\right\rangle \in \Gamma$. A curb set is a product set $X=\times_{i \in N} X_{i}$, where

- for each $i \in N, X_{i} \subseteq A_{i}$ is a nonempty, compact set of pure strategies;

- for each $i \in N$ and each belief $\alpha_{-i}$ of player $i$ with support in $X_{-i}$, the set $X_{i}$ contains all best responses of player $i$ against his belief:

$$
\forall i \in N, \forall \alpha_{-i} \in \times_{j \in N \backslash\{i\}} \Delta\left(X_{j}\right): \quad B R_{i}\left(\alpha_{-i}\right) \subseteq X_{i}
$$

A curb set $X$ is minimal if no curb set is a proper subset of $X$. Similarly, a prep set is a product set $X=\times_{i \in N} X_{i}$, where 
- for each $i \in N, X_{i} \subseteq A_{i}$ is a nonempty, compact set of pure strategies;

- for each $i \in N$ and each belief $\alpha_{-i}$ of player $i$ with support in $X_{-i}$, the set $X_{i}$ contains at least one best response of player $i$ against his belief:

$$
\forall i \in N, \forall \alpha_{-i} \in \times_{j \in N \backslash\{i\}} \Delta\left(X_{j}\right): \quad B R_{i}\left(\alpha_{-i}\right) \cap X_{i} \neq \emptyset .
$$

A prep set $X$ is minimal if no prep set is a proper subset of $X$.

\section{Sufficient conditions for coincidence}

In this section, we show that some simple conditions are sufficient for the collection of minimal prep sets and the collection of pure Nash equilibria to coincide in a class of games. This statement is intuitively clear, but since we are comparing set-valued solutions with point-valued solutions, let us define the coincidence formally: in a game $G=\left\langle N,\left(A_{i}\right)_{i \in N},\left(u_{i}\right)_{i \in N}\right\rangle \in \Gamma$, the collection of minimal prep sets and the collection of pure Nash equilibria coincide if for each minimal prep set $X=\times_{i \in N} X_{i}$ of $G$ and each player $i \in N$, there is a pure strategy $a_{i} \in A_{i}$ such that $X_{i}=\left\{a_{i}\right\}$ and $a=\left(a_{i}\right)_{i \in N}$ is a pure Nash equilibrium. (Its 'converse', namely that for each pure Nash equilibrium $a=\left(a_{i}\right)_{i \in N} \in A$, the product set $\times_{i \in N}\left\{a_{i}\right\}$ is a minimal prep set, is true by definition.)

If $\Gamma^{\prime} \subseteq \Gamma$ is a class of games, we say that the collection of minimal prep sets and the collection of pure Nash equilibria coincide on $\Gamma^{\prime}$ if they coincide for each game $G \in \Gamma^{\prime}$.

For a game $G=\left\langle N,\left(A_{i}\right)_{i \in N},\left(u_{i}\right)_{i \in N}\right\rangle \in \Gamma$, we will sometimes wish to restrict players' pure strategies to a product set $B=\times_{i \in N} B_{i} \subseteq A$. The game's payoffs are trivially obtained by restricting the payoff functions $\left(u_{i}\right)_{i \in N}$ to $B$. With a slight abuse of notation (letting the domain of payoffs be implicit), this subgame of $G$ is denoted by $\left\langle N,\left(B_{i}\right)_{i \in N},\left(u_{i}\right)_{i \in N}\right\rangle$.

Let us formulate the conditions under which we will establish coincidence. A class of games $\Gamma^{\prime} \subseteq \Gamma$ :

- is closed w.r.t. subgames if for each game $G=\left\langle N,\left(A_{i}\right)_{i \in N},\left(u_{i}\right)_{i \in N}\right\rangle \in \Gamma^{\prime}$ and each nonempty product set $B=\times_{i \in N} B_{i} \subseteq A$ of compact action sets $B_{i} \subseteq A_{i}$, also $\left\langle N,\left(B_{i}\right)_{i \in N},\left(u_{i}\right)_{i \in N}\right\rangle \in \Gamma^{\prime}$;

- is closed w.r.t. minimal prep sets if for each game $G=\left\langle N,\left(A_{i}\right)_{i \in N},\left(u_{i}\right)_{i \in N}\right\rangle \in \Gamma^{\prime}$ and each minimal prep set $B=\times_{i \in N} B_{i} \subseteq A$, also $\left\langle N,\left(B_{i}\right)_{i \in N},\left(u_{i}\right)_{i \in N}\right\rangle \in \Gamma^{\prime}$;

- has the pure Nash property if each game $G \in \Gamma^{\prime}$ has a pure Nash equilibrium.

Clearly, if $\Gamma^{\prime}$ is closed w.r.t. subgames, it is closed w.r.t. minimal prep sets. The set of matrix games (i.e., finite, two-player zero-sum games) is closed w.r.t. subgames and in particular w.r.t. minimal prep sets, but does not have the pure Nash property. The set of best-response potential games with a finite pure strategy space (see Sect. 5.1) has the pure Nash property and is closed w.r.t. minimal prep sets, but is not closed w.r.t. subgames.

Proposition 3.1 If $\Gamma^{\prime} \subseteq \Gamma$ has the pure Nash property and is closed w.r.t. minimal prep sets, then the set of pure Nash equilibria and the collection of minimal prep sets coincide on $\Gamma^{\prime}$. 
Proof Let $G=\left\langle N,\left(A_{i}\right)_{i \in N},\left(u_{i}\right)_{i \in N}\right\rangle \in \Gamma^{\prime}$. By definition, for each pure Nash equilibrium $a \in A, \times_{i \in N}\left\{a_{i}\right\}$ is a minimal prep set. Conversely, let $B=\times_{i \in N} B_{i}$ be a minimal prep set of $G$. Since $\Gamma^{\prime}$ is closed w.r.t. minimal prep sets, also $\left\langle N,\left(B_{i}\right)_{i \in N},\left(u_{i}\right)_{i \in N}\right\rangle \in$ $\Gamma^{\prime}$. By the pure Nash property, $\left\langle N,\left(B_{i}\right)_{i \in N},\left(u_{i}\right)_{i \in N}\right\rangle$ has a pure Nash equilibrium $b=\left(b_{i}\right)_{i \in N} \in B$ and hence minimal prep set $\times_{i \in N}\left\{b_{i}\right\}$. Since $B$ is a prep set of $G$, it follows that $\times_{i \in N}\left\{b_{i}\right\}$ is a minimal prep set of the original game $G$. Moreover, as it is a singleton set, $b$ is a Nash equilibrium of $G$. Since $\times_{i \in N}\left\{b_{i}\right\} \subseteq B$ and $B$ is a minimal prep set of $G$, it follows that $\times_{i \in N}\left\{b_{i}\right\}=B$ : the minimal prep set $B$ corresponds with a pure Nash equilibrium.

Although Proposition 3.1 is relatively straightforward, its power comes from the fact that it is applicable - as is shown in the following sections-in many well-known and practically relevant classes of games. It applies in particular to-and is arguably intuitively most appealing for-a class of games which is closed w.r.t. subgames. This is regularly the case if the games are defined by common types of strategic interactions: subgames of zero-sum games are zero-sum, subgames of congestion games, where players choose among different roads/facilities, are congestion games, etc. Nevertheless, many interesting classes of games with the pure Nash property are not closed w.r.t. subgames, even though they are closed w.r.t. minimal prep sets. Specific examples include the games with strategic complementarities in Sect. 4.2 and the best-response potential games in Sect. 5.1.

A pure saddle-point theorem of Shapley (1964, pp. 6-7) serves as a first illustration of the use of Proposition 3.1. Other economically relevant applications are provided in later sections.

Proposition 3.2 Let $\Gamma^{\prime}$ be the set of finite two-person zero-sum games

$$
G=\left\langle\{1,2\},\left(A_{1}, A_{2}\right),\left(u_{1},-u_{1}\right)\right\rangle
$$

in which each $2 \times 2$ subgame (a subgame in which both players have exactly two pure strategies) has a pure saddle point/Nash equilibrium ${ }^{2}$. For each game $G \in \Gamma^{\prime}$, the set of pure saddle points/Nash equilibria and the collection of minimal prep sets coincide.

Proof Let $G \in \Gamma^{\prime}$ and let $H$ be a subgame of $G$. Since each $2 \times 2$ subgame of $H$ is a $2 \times 2$ subgame of $G$, it follows that all $2 \times 2$ subgames of $H$ have a pure saddle point. Conclude that $\Gamma^{\prime}$ is closed w.r.t. subgames. Moreover, $\Gamma^{\prime}$ has the pure Nash property by Thm. 2.1 of Shapley (1964). The result now follows from Proposition 3.1.

\section{Strategic complementarities}

Well-known existence results for Nash equilibria in supermodular games or games with strategic complementarities rely on monotonicity properties of the best-reply correspondence. The theory was initiated by Topkis (1978) and has been successfully applied to a wide range of economic models; the reader is referred to, for instance,

\footnotetext{
2 This assumption holds vacuously for finite two-person zero-sum games in which some player has only one pure strategy: there are no $2 \times 2$ subgames! Hence, such games are included in $\Gamma^{\prime}$.
} 
Milgrom and Roberts (1990) or the book of Topkis (1998). This section relies on a general existence result by Zhou's (1994).

\subsection{Lattices and order}

A partially ordered set $(S, \leq)$ is a set $S$ with a binary relation $\leq$ which is:

(1) reflexive: $\forall x \in S: x \leq x$,

(2) antisymmetric: $\forall x, y \in S$ : if $x \leq y$ and $y \leq x$, then $x=y$,

(3) transitive: $\forall x, y, z \in S$ : if $x \leq y$ and $y \leq z$, then $x \leq z$.

If the partial order $\leq$ is complete $(\forall x, y \in S: x \leq y$ or $y \leq x)$, it is a linear order.

Let $(S, \leq)$ be a partially ordered set and let $T \subseteq S$. An element $x \in S$ is a lower bound of $T$ if $x \leq y$ for all $y \in T$ and an upper bound of $T$ if $y \leq x$ for all $y \in T$. If it exists, the least upper bound of $T$ is called the supremum $\sup (T)$ of $T$ in $S$ and the greatest lower bound of $T$ is called the infimum $\inf (T)$ of $T$ in $S$. A lattice is a partially ordered set $(S, \leq)$ that contains the infimum $x \wedge y=\inf \{x, y\}$ and supremum $x \vee y=\sup \{x, y\}$ of each pair of elements $x, y \in S$. The lattice is complete if, for all nonempty subsets $T \subseteq S$ : $\inf (T)$ and $\sup (T)$ exist.

$(T, \leq)$ is a sublattice of lattice $(S, \leq)$ if $T \subseteq S$ is closed under $\wedge$ and $\vee$, i.e., if it is a lattice with the same join and meet relations as $S$. As above, this sublattice is complete if, for all nonempty subsets $U \subseteq T: \inf (U) \in T$ and $\sup (U) \in T$.

An interval $[x, y]$ in $(S, \leq)$ is the set $\{z \in S \mid x \leq z \leq y\}$. For $x \in S$, we denote $(-\infty, x]=\{z \in S \mid z \leq x\}$ and $[x, \infty)=\{z \in S \mid x \leq z\}$. The interval topology on a lattice $(S, \leq)$ is the topology for which all closed sets are intersections or finite unions of intervals of the form $S,(-\infty, x]$, and $[x, \infty)$, where $x \in S$. By the Frink-Birkhoff theorem (Birkhoff 1967), a lattice is complete if and only if it is compact in its interval topology. Hence, any sublattice of a complete lattice is complete if and only if it is closed in its interval topology.

A note of caution: a subset of $(S, \leq)$ that is a complete lattice in its own right may not be a complete sublattice of $(S, \leq)$. Milgrom and Roberts (1990, p. 1260) give enlightening examples. For instance, the set $T=[0,1) \cup\{2\}$ is a complete lattice under its standard order. In this case $\sup [0,1)=2 \in T$. It is not a complete sublattice of $[0,2]$, where $\sup [0,1)=1 \notin T$.

Let $(S, \leq)$ and $(T, \precsim)$ be two lattices. A correspondence $\varphi: S \rightarrow T$ is ascending if, for all $s, s^{\prime} \in S$ with $s \leq s^{\prime}$, all $t \in \varphi(s)$ and $t^{\prime} \in \varphi\left(s^{\prime}\right)$, it holds that $t \wedge t^{\prime} \in \varphi(s), t \vee t^{\prime} \in$ $\varphi\left(s^{\prime}\right)$.

\subsection{Games with ascending best replies}

As stated, well-known existence results for Nash equilibria rely on the best-response correspondence being ascending. This can be derived from other assumptions under names like supermodularity/strategic complementarity/increasing differences, but the key to the result is always the monotonicity of best replies. Therefore, we state our result in terms of ascending best replies and refer to, for instance, Zhou's (1994) for a clear account on how to achieve it from other conditions. 
We use the general existence result by Zhou's (1994) with the only modification that we assume all action sets $A_{i}$ to be linearly ordered, rather than just a lattice. In most applications (see Milgrom and Roberts 1990; Topkis 1998), this assumption is satisfied. Often, for instance, $A_{i}$ is a set of real numbers with its usual order.

Recall that a function $f: A \rightarrow \mathbb{R}$ on a topological space $A$ is upper semicontinuous (u.s.c.) if its upper contour sets are closed:

$$
\forall r \in \mathbb{R}:\{a \in A \mid f(a) \geq r\} \text { is closed. }
$$

Proposition 4.1 Let $\Gamma^{A S C}$ be the set of strategic games $G=\left\langle N,\left(A_{i}\right)_{i \in N},\left(u_{i}\right)_{i \in N}\right\rangle \in$ $\Gamma$ satisfying the following conditions:

(ASC1) For each $i \in N$, there is a linear order $\leq_{i}$ on $A_{i}$ such that $A_{i}$ is compact in a topology $\tau_{i}$ equal to or finer than the interval topology. ${ }^{3}$

(ASC2) For each $i \in N, u_{i}$ is upper semicontinuous on $A_{i}$ in the topology $\tau_{i}$.

(ASC3) For each $i \in N$, the best-response correspondence $B R_{i}: A_{-i} \rightarrow A_{i}$ is ascending. ${ }^{4}$

For each game $G \in \Gamma^{A S C}$ the collection of minimal prep sets and pure Nash equilibria coincide.

Proof $\Gamma^{A S C}$ HAS THE PURE NASH PROPERTY: All games in $\Gamma^{A S C}$ satisfy Zhou's (1994) sufficient conditions for the existence of pure Nash equilibria: $A$ is a complete lattice; for each $a \in A$, the set $B R(a)=\times_{i \in N} B R_{i}\left(a_{-i}\right)$ is a nonempty, closed sublattice of $A$ by (ASC1) and (ASC2); the best response correspondence is ascending. $\Gamma^{A S C}$ IS CLOSED W.R.T. MINIMAL PREP SETS: Let $G=\left\langle N,\left(A_{i}\right)_{i \in N},\left(u_{i}\right)_{i \in N}\right\rangle \in$ $\Gamma^{A S C}$ and let $B=\times_{i \in N} B_{i} \subseteq A$ be a minimal prep set of $G$. To show: $H=$ $\left\langle N,\left(B_{i}\right)_{i \in N},\left(u_{i}\right)_{i \in N}\right\rangle \in \Gamma^{A S C}$.

(i) Restricting the linear order $\leq_{i}$ on $A_{i}$ to $B_{i}$, we see that $\left(B_{i}, \leq_{i}\right)$ is linearly ordered.

(ii) By definition of a minimal prep set, $B_{i}$ is compact in $\tau_{i}$, which is equal to or finer than the interval topology on $A_{i}$. Hence, the same holds for the topology restricted to $B_{i}$, the usual subspace topology.

(iii) Since $u_{i}$ is upper semicontinuous in the topology $\tau_{i}$ on $A_{i}$, it remains so on $B_{i}$.

(iv) Since $B$ is a minimal prep set, the best-response correspondence $B R_{i}^{H}(\cdot)$ of the subgame $H$ is given by

$$
B R_{i}^{H}(\cdot)=B_{i} \cap B R_{i}^{G}(\cdot),
$$

the-by definition of a minimal prep set-nonempty intersection of the bestresponse correspondence of the original game $G$ and $i$ 's component $B_{i}$ of the minimal prep set. Since $B R_{i}^{G}(\cdot)$ is ascending and $B_{i}$ is a lattice given its linear order $\leq_{i}$, it follows that $B R_{i}^{H}(\cdot)$ is ascending.

\footnotetext{
3 Topology $\tau_{i}$ is finer than the interval topology if every open set in the interval topology is open in $\tau_{i}$.

4 As usual, $A_{-i}$ is the direct product compact lattice whose product order $\leq_{-i}$ is such that for all $a_{-i}=$ $\left(a_{j}\right)_{j \in N \backslash\{i\}}$ and $b_{-i}=\left(b_{j}\right)_{j \in N \backslash\{i\}}$ in $A_{-i}: a_{-i} \leq_{-i} b_{-i}$ iff $a_{j} \leq_{j} b_{j}$ for all $j \in N \backslash\{i\}$.
} 
Fig. 1 Supermodular games: not closed w.r.t. subgames

Fig. 2 Supermodular games: non-linear orders

\begin{tabular}{c|c|c|c|}
\multicolumn{1}{c}{} & \multicolumn{1}{c}{0} & \multicolumn{1}{c}{1} & \multicolumn{1}{c}{2} \\
\cline { 2 - 4 } 0 & 2,2 & 2,0 & 2,0 \\
\cline { 2 - 4 } 1 & 0,2 & $1,-1$ & $-1,1$ \\
\cline { 2 - 4 } 2 & 0,2 & $-1,1$ & $1,-1$ \\
\cline { 2 - 4 } & &
\end{tabular}

\begin{tabular}{c|c|c|c|c|}
\multicolumn{1}{c}{} & \multicolumn{1}{c}{0} & 1 & 2 & 3 \\
\cline { 2 - 5 } 0 & 2,2 & 0,0 & 0,0 & 0,0 \\
\cline { 2 - 5 } 1 & 0,0 & 1,0 & 0,1 & 0,0 \\
\cline { 2 - 5 } 2 & 0,0 & 0,1 & 1,0 & 0,0 \\
\cline { 2 - 5 } 3 & 0,0 & 0,0 & 0,0 & 2,2 \\
\cline { 2 - 5 } & & &
\end{tabular}

Combining observations $(i)$ to $(i v)$, it follows that $\left\langle N,\left(B_{i}\right)_{i \in N},\left(u_{i}\right)_{i \in N}\right\rangle \in \Gamma^{A S C}$, i.e., $\Gamma^{A S C}$ is closed w.r.t. minimal prep sets. The result now follows from Proposition 3.1.

Remarks (i) The set $\Gamma^{A S C}$ in Proposition 4.1 is not closed w.r.t. subgames: in the two-player game in Fig. 1, each player's action 0 is strictly dominant.

Hence, the best-response correspondences are constant and in particular ascending in the usual order on $\{0,1,2\}$ : the game belongs to $\Gamma^{A S C}$. But the subgame on $\{1,2\} \times\{1,2\}$ is not in $\Gamma^{A S C}$ : it has no pure Nash equilibrium.

(ii) Proposition 4.1 does not hold if the assumption that each action set $A_{i}$ is linearly ordered is relaxed to assuming that there is an order $\leq_{i}$ on $A_{i}$ such that $\left(A_{i}, \leq_{i}\right)$ is a complete lattice: the associated class of games is not closed w.r.t. minimal prep sets. Consider the two-player game in Fig. 2. Define, for each player $i$, the partial order $\leq_{i}$ on $A_{i}=\{0,1,2,3\}$ with $0 \leq_{i} 1 \leq_{i} 3$ and $0 \leq_{i} 2 \leq_{i} 3$, but which does not compare 1 and 2 . Then $\left(A_{i}, \leq_{i}\right)$ is a complete lattice. The players' best-response correspondences are:

$B R_{1}\left(a_{2}\right)=\left\{a_{2}\right\}$ for all $a_{2} \in\{0,1,2,3\}$ and $B R_{2}\left(a_{1}\right)= \begin{cases}\{0\} & \text { if } a_{1}=0 \\ \{2\} & \text { if } a_{1}=1 \\ \{1\} & \text { if } a_{1}=2 \\ \{3\} & \text { if } a_{1}=3\end{cases}$

Hence, the best-response correspondences $B R_{1}$ and $B R_{2}$ are ascending. The product set $\{1,2\} \times\{1,2\}$ is a minimal prep set of the game. In the subgame restricted to these action profiles, we still have that the best response correspondence is (trivially) ascending with respect to the product order induced by the restriction of $\leq_{i}$ to $\{1,2\}$. But $\left(\{1,2\}, \leq_{i}\right)$ is not a lattice: $1 \wedge 2$ and $1 \vee 2$ do not exist. Notice, indeed, that this subgame does not have a pure Nash equilibrium. 
(iii) Recall that a (necessarily pure) Nash equilibrium is strict if each player has a unique best response to the choices of the remaining players. In generic finite games belonging to the class of games with ascending best replies studied above, also the collection of minimal curb sets coincides with the collection of pure and strict Nash equilibria: ${ }^{5}$ take the subclass of games where all payoffs are distinct (its relative complement is definitely a set of measure zero). Clearly, all pure Nash equilibria are strict in such games. Any minimal curb set $B$ is a prep set, hence contains a minimal prep set, which is a singleton set consisting of one pure Nash equilibrium. This singleton set is curb too, so $B$ is a Nash equilibrium! This result has an important impact for convergence of adjustment processes: it is within minimal curb sets that many intuitive models of strategic adjustment settle down; cf. Young (1993, 1998); Hurkens (1995); Kosfeld et al. (2002). Hence, in generic, finite games in $\Gamma^{A S C}$, these processes converge toward a strict Nash equilibrium.

Although of interest in its own right, many classes of games have additional structure, making them congeneric. To illustrate this point, the next subsection describes a subclass of supermodular games in which minimal prep sets give sharp predictions, whereas minimal curb sets have no cutting power whatsoever and simply consist of the entire strategy space.

\subsection{A class of coordination games}

By Proposition 4.1, minimal prep sets have substantial cutting power in a very general class of supermodular games. However, one can easily construct plausible subclasses of such games where minimal curb sets have no cutting power. We give a simple example.

Consider a two-player coordination game where the players find each other if they choose close-by alternatives. Formally, consider the game $G=\left\langle\{1,2\},\left(A_{1}, A_{2}\right)\right.$, $\left.\left(u_{1}, u_{2}\right)\right\rangle$ where

$$
A_{1}=A_{2}=\{0,1, \ldots, k\} \text { for some } k \in \mathbb{N}
$$

and for each pair of alternatives $\left(a_{1}, a_{2}\right) \in A_{1} \times A_{2}$ :

$$
u_{1}\left(a_{1}, a_{2}\right)=u_{2}\left(a_{1}, a_{2}\right)= \begin{cases}1 & \text { if }\left|a_{1}-a_{2}\right| \leq 1, \\ 0 & \text { otherwise }\end{cases}
$$

i.e., the players choose a location $0, \ldots, k$ and are rewarded ('find each other') if they choose identical or neighboring locations.

Proposition 4.2 In a two-player coordination game $G=\left\langle\{1,2\},\left(A_{1}, A_{2}\right),\left(u_{1}, u_{2}\right)\right\rangle$ as in (1) and (2), the following hold:

\footnotetext{
5 We are grateful to an anonymous referee for suggesting this shorter version of our original proof.
} 
(a) the collections of pure Nash equilibria and minimal prep sets coincide;

(b) the collection of pure Nash equilibria is

$$
\left\{\left(a_{1}, a_{2}\right) \in A_{1} \times A_{2}:\left|a_{1}-a_{2}\right| \leq 1\right\} ;
$$

(c) the unique (hence minimal) curb set is $A_{1} \times A_{2}$.

Proof (a) Endowing the action space $A_{i}=\{0,1, \ldots, k\}$ of player $i \in\{1,2\}$ with its standard order, the game is easily seen to belong to the class of games with ascending best responses in Proposition 4.1, so that pure Nash equilibria and minimal prep sets indeed coincide.

(b) Follows easily from (2).

(c) Let $X=X_{1} \times X_{2}$ be a curb set of $G$. Fix a player $i \in\{1,2\}$. By (2), it follows that if $a_{i} \in X_{i}$, then $\left\{a_{i}-1, a_{i}, a_{i}+1\right\} \cap\{0,1, \ldots, k\} \subseteq X_{j}$ for $j \neq i$ : player $j$ 's component of the curb set contains not only $a_{i}$, but also the neighboring actions. The only set with this property is $A_{1} \times A_{2}$.

\section{Potential games}

\subsection{Generalized ordinal and best-response potential games}

Monderer and Shapley (1996) define four classes of potential games, in increasing order of generality: exact, weighted, ordinal, and generalized ordinal potential games. These games have applications to, for instance, congestion models (Rosenthal 1973) and oligopoly models (Slade 1994). All finite potential games in Monderer and Shapley (1996) have the following finite improvement property: start with an arbitrary strategy profile. Each time, let a player that can benefit from unilateral deviation switch to a better strategy. Under the finite improvement property, this process eventually ends, obviously in a Nash equilibrium. Voorneveld (2000) introduces best-response potential games that allow infinite improvement paths by imposing restrictions only on paths in which players that can improve actually deviate to a best response. These games include the best-response potential games of Morris and Ui (2004, p. 264, after Def. 6). Formally, a game $G=\left\langle N,\left(A_{i}\right)_{i \in N},\left(u_{i}\right)_{i \in N}\right\rangle$ is

- a generalized ordinal potential game if there is a function $P: A \rightarrow \mathbb{R}$ such that, for each player $i \in N$, each strategy profile $a_{-i} \in A_{-i}$ of his fellow players, and each pair of strategies $a_{i}, b_{i} \in A_{i}$ :

$$
u_{i}\left(a_{i}, a_{-i}\right)-u_{i}\left(b_{i}, a_{-i}\right)>0 \Rightarrow P\left(a_{i}, a_{-i}\right)-P\left(b_{i}, a_{-i}\right)>0 .
$$

- a best-response potential game if there is a function $P: A \rightarrow \mathbb{R}$ such that, for each player $i \in N$ and each strategy profile $a_{-i} \in A_{-i}$ of his fellow players:

$$
\arg \max _{a_{i} \in A_{i}} u_{i}\left(a_{i}, a_{-i}\right)=\arg \max _{a_{i} \in A_{i}} P\left(a_{i}, a_{-i}\right) .
$$

The function $P$ is called a (generalized ordinal or best-response) potential. 
There is no logical dependence between (3) and (4): Examples 4.1 and 4.2 in Voorneveld (2000) indicate that there are generalized ordinal potential games which are not best-response potential games, and conversely, that there are best-response potential games which are not generalized ordinal potential games.

Proposition 5.1 Let $\Gamma^{G O P}$ and $\Gamma^{B R P}$ be the set of games with compact strategy spaces and an upper semicontinuous generalized ordinal or best-response potential, respectively. For each $G \in \Gamma^{G O P} \cup \Gamma^{B R P}$, the set of pure Nash equilibria and the collection of minimal prep sets coincide.

Proof $\Gamma^{G O P}$ IS CLOSED W.R.T. SUBGAMES: Simply restrict the domain of the potential function.

$\Gamma^{B R P}$ IS CLOSED W.R.T. MINIMAL PREP SETS: Let $G=\left\langle N,\left(A_{i}\right)_{i \in N},\left(u_{i}\right)_{i \in N}\right\rangle \in$ $\Gamma^{B R P}$ have u.s.c. best-response potential $P$ and assume $B=\times_{i \in N} B_{i}$ is a minimal prep set of $G$. For each player $i \in N$ and each strategy profile $a_{-i} \in \times_{j \in N \backslash\{i\}} B_{j}$,

$$
\emptyset \neq \arg \max _{a_{i} \in A_{i}} u_{i}\left(a_{i}, a_{-i}\right) \cap B_{i}=\arg \max _{a_{i} \in A_{i}} P\left(a_{i}, a_{-i}\right) \cap B_{i},
$$

where the inequality follows by definition of a prep set and the equality follows from (4). Hence, also the game $H=\left\langle N,\left(B_{i}\right)_{i \in N},\left(u_{i}\right)_{i \in N}\right\rangle$ is a best-response potential game, with a best response potential obtained from $P$ by restricting its domain. Its strategy spaces $\left(B_{i}\right)_{i \in N}$ are compact by definition of a prep set and $P$ remains u.s.c. in the subspace topology. Conclude that $H \in \Gamma^{B R P}$.

$\Gamma^{G O P}$ AND $\Gamma^{B R P}$ HAVE THE PURE NASH PROPERTY: Since $A$ is compact in the product topology and each $G \in \Gamma^{G O P} \cup \Gamma^{B R P}$ has an upper semicontinuous potential $P$, the potential achieves a maximum. By (3) or (4), such a maximum is a pure Nash equilibrium.

The result now follows from Proposition 3.1.

Remarks (i) Endowing $A$ with the discrete topology, the conclusion of Proposition 5.1 applies in particular to finite generalized ordinal/best-response potential games.

(ii) As opposed to the set of generalized ordinal potential games, the set of bestresponse potential games is not closed w.r.t. subgames: The two-player game to the left in Fig. 3 has a best-response potential (to the right). The subgame with action space $\{T, B\} \times\{M, R\}$ is not a best-response potential game: such a potential would have to satisfy

$$
P(T, M)<P(T, R)<P(B, R)<P(B, M)<P(T, M),
$$

a contradiction.

Fig. 3 Best-response potential games: not closed w.r.t. subgames

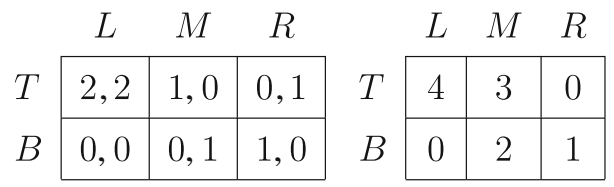


Fig. 4 A pseudo-potential game

\begin{tabular}{c|c|c|c|c|}
\multicolumn{1}{c}{$A$} & \multicolumn{1}{c}{$B$} & \multicolumn{1}{c}{$C$} & \multicolumn{1}{c}{$D$} \\
\cline { 2 - 5 }$T$ & $1,-1$ & $-1,1$ & 1,1 & $-2,-2$ \\
\cline { 2 - 5 }$B$ & $-1,1$ & $1,-1$ & $-2,-2$ & 1,1 \\
\cline { 2 - 5 } & &
\end{tabular}

\begin{tabular}{c|c|c|c|c|}
\multicolumn{1}{c}{$A$} & \multicolumn{1}{c}{$B$} & $C$ & \multicolumn{1}{c}{$D$} \\
\hline \multirow{2}{*}{$T$} & 1 & 0 & 2 & 0 \\
\cline { 2 - 5 }$B$ & 0 & 1 & 0 & 2 \\
\hline
\end{tabular}

(iii) The assumption that the game needs to have an u.s.c. potential is not an innocuous one. Voorneveld (1997, pp. 167-168) gives an example of an ordinal potential game with compact strategy spaces and continuous payoff functions for which no potential achieves a maximum and which, consequently, has no u.s.c. potential.

(iv) The conclusion of Proposition 5.1 does not hold for the pseudo-potential games recently introduced by Dubey et al. (2006). Formally, a game $G=\left\langle N,\left(A_{i}\right)_{i \in N}\right.$, $\left.\left(u_{i}\right)_{i \in N}\right\rangle$ is a pseudo-potential game if there is a function $P: A \rightarrow \mathbb{R}$ such that, for each player $i \in N$ and each strategy profile $a_{-i} \in A_{-i}$ of his fellow players:

$$
\arg \max _{a_{i} \in A_{i}} u_{i}\left(a_{i}, a_{-i}\right) \supseteq \arg \max _{a_{i} \in A_{i}} P\left(a_{i}, a_{-i}\right) .
$$

The two-player game to the left in Fig. 4 has a pseudo-potential (to the right). But its pure Nash equilibria and minimal prep sets do not coincide, since $\{T, B\} \times$ $\{A, B\}$ is a minimal prep set. The game has neither a generalized ordinal nor a best-response potential function $P$, which by definition would have to satisfy:

$$
P(T, A)<P(T, B)<P(B, B)<P(B, A)<P(T, A),
$$

a contradiction.

(v) As we showed for supermodular games, also in generic finite generalized ordinal/best-response potential games, the collection of minimal curb sets and the collection of pure (and strict) Nash equilibria coincide. Again, we note that in such games, adjustment processes as in Young (1993, 1998); Hurkens (1995), and Kosfeld et al. (2002) converge toward a strict Nash equilibrium.

\subsection{Minority games}

Above, we saw that in large classes of potential games, minimal prep sets have substantial cutting power, yielding equilibrium predictions. On the one hand, we have shown that in finite generic potential games, the collection of minimal curb sets and the collection of minimal prep sets coincide. On the other hand, just as for supermodular games, in economically relevant subclasses of these games, minimal curb sets have no cutting power whatsoever. As an example, this section considers so-called minority games, a type of congestion problems introduced by Challet and Zhang (1997) and inspired by the El Farol Bar problem of Arthur (1994). See Moro (2003) for an introductory overview, Challet et al. (2004) for a book containing many of the path-breaking papers within the physics literature and applications to phenomena in financial markets, and Coolen (2005) for a thorough mathematical treatment.

A minority game is a congestion problem where players aim to avoid crowds and prefer choosing the minority alternative. It has an odd number of players: $N=$ $\{1, \ldots, 2 k+1\}$ for some $k \in \mathbb{N}$. Each player $i \in N$ chooses among two actions: $A_{i}=\{-1,+1\}$ for all $i \in N$. For each action $s \in\{-1,+1\}$ there is a function 


$$
f_{s}:\{1, \ldots, 2 k+1\} \rightarrow \mathbb{R}
$$

where, for each $m \in\{1, \ldots, 2 k+1\}$, the number $f_{s}(m) \in \mathbb{R}$ specifies the utility/payoff to a player choosing $s$ if the total number of players choosing $s$ equals $m$. The payoff/utility function $u_{i}: A \rightarrow \mathbb{R}$ of player $i \in N$ is consequently defined as follows:

$$
\forall a=\left(a_{j}\right)_{j \in N} \in A: \quad u_{i}(a)=f_{a_{i}}\left(\left|\left\{j \in N: a_{j}=a_{i}\right\}\right|\right) .
$$

Characteristic for a minority game is the assumption that unilateral deviation from a majority to a minority pays off:

$$
\forall s, t \in\{-1,+1\}, s \neq t, \forall m \in\{k+2, \ldots, 2 k+1\}: \quad f_{s}(m)<f_{t}(2 k+2-m) .
$$

Example 5.2 Challet and Zhang (1997, p. 408) who introduce minority games, initially assign payoff one to each member of the minority, and payoff zero to each member of a majority:

$$
f_{-1}(m)=f_{+1}(m)= \begin{cases}1 & \text { if } m \in\{1, \ldots, k\}, \\ 0 & \text { if } m \in\{k+1, \ldots, 2 k+1\} .\end{cases}
$$

In a variant (Challet and Zhang 1997, p. 411), they suggest payoffs giving zero reward to majority members and positive payoffs to minorities, favoring small ones:

$$
f_{-1}(m)=f_{+1}(m)= \begin{cases}|N| / m-2 & \text { if } m \in\{1, \ldots, k\}, \\ 0 & \text { if } m \in\{k+1, \ldots, 2 k+1\} .\end{cases}
$$

Given an action profile $a=\left(a_{i}\right)_{i \in N}$, the minority alternative is -1 if $\sum_{i \in N} a_{i}>0$ and +1 if $\sum_{i \in N} a_{i}<0$. Other frequently occurring payoff functions (Moro 2003) assign to player $i$ a payoff given by $-a_{i} g\left(\sum_{j \in N} a_{j}\right)$, where $g$ is an odd function, i.e., $g(x)=-g(-x)$, with $g(x)>0$ if $x>0$. In particular, common examples include

$$
g(x)=x /|N| \text { and } g(x)=\operatorname{sgn}(x),
$$

where the sign function is defined for each $x \in \mathbb{R}$ as:

$$
\operatorname{sgn}(x)=\left\{\begin{aligned}
-1 & \text { if } \quad x<0 \\
0 & \text { if } \quad x=0 \\
+1 & \text { if } \quad x>0
\end{aligned}\right.
$$

In our notation:

$$
f_{-1}(m)=f_{+1}(m)=g(2(k-m)+1) .
$$

As is seen from the examples, the payoff functions to the two alternatives are traditionally assumed to be identical: $f_{-1}=f_{+1}$. We relax this assumption by only requiring

$$
f_{-1}(k+1)=f_{+1}(k+1)
$$


Proposition 5.3 In a minority game G (in particular, under assumptions (5) and (10)) with $2 k+1$ players, the following holds:

(a) $G$ is a potential game, so its pure Nash equilibria and minimal prep sets coincide;

(b) A pure strategy profile $a \in A$ is a Nash equilibrium if and only if there is an alternative $s \in\{-1,+1\}$ chosen by exactly $k$ players, i.e., $\left|\left\{i \in N: a_{i}=s\right\}\right|=k$;

(c) The unique minimal curb set of $G$ is the entire pure strategy space.

Proof (a) $G$ is a congestion game as in Rosenthal (1973) and hence a (finite exact) potential game (Monderer and Shapley 1996, Thm. 3.1). By Proposition 5.1, its pure Nash equilibria and minimal prep sets coincide.

(b) Fix a pure strategy profile. The number of players is odd, so some option $s \in$ $\{-1,+1\}$ is chosen by a majority of at least $k+1$ players. If the majority has $k+2$ or more players, (5) implies that a majority member can unilaterally deviate to the other option and achieve a strictly higher payoff. Thus, the strategy profiles in Proposition 5.3(b) are the only candidates for pure Nash equilibria. They are indeed equilibria: by (5), a minority member never has an incentive to deviate and join a majority. Next, let $s \in\{-1,+1\}$ be the alternative chosen by the $k+1$ majority members. If a majority member deviates to $t \neq s$, his payoff changes from $f_{s}(k+1)$ to $f_{t}(k+1)$. By $(10)$, these payoffs are the same. Conclude: the profiles in Proposition 5.3(b) are indeed the game's pure Nash equilibria.

(c) Let $B=\times_{i \in N} B_{i}$ be a minimal curb set of $G$. At least one of the sets $\{i \in N$ : $\left.-1 \in B_{i}\right\}$ and $\left\{i \in N:+1 \in B_{i}\right\}$, w.l.o.g. the latter, contains $k+1$ or more players. Let $I \subseteq\left\{i \in N:+1 \in B_{i}\right\}$ have $k+1$ members and choose $b \in B$ with $b_{i}=+1$ if $i \in I$. For each of the $k$ remaining players $j \in N \backslash I$, the unique best reply to $b_{-j}$ is -1 , so $-1 \in B_{j}$. When the $k+1$ players in $I$ play +1 and the $k$ players in $N \backslash I$ play -1 , the members of $I$ are indifferent between their actions by (10), so by definition of a minimal curb set, $B_{i}=\{-1,+1\}$ for all $i \in I$. Similarly, choose $b^{\prime} \in B$ with $b_{i}^{\prime}=-1$ if $i \in I$. For each of the $k$ remaining players $j \in N \backslash I$, the unique best reply to $b_{-j}^{\prime}$ is +1 , so $+1 \in B_{j}$. We already had that $-1 \in B_{j}$, so $B_{j}=\{-1,+1\}$. Hence, $B_{i}=\{-1,+1\}$ for all $i \in N$.

\subsection{An extension to the Quint-Shubik congestion model}

Monderer and Shapley (1996, Thm. 3.2) show that each finite exact potential game is isomorphic to a congestion game as defined in Rosenthal (1973). In these games, players choose subsets of facilities from a common pool. The payoff associated with each facility is a function only of the number of players using it. Quint and Shubik (1994) and-as a special case-Milchtaich (1996) considered a different class of congestion games by allowing payoffs to be player-dependent. In general, these games do not admit a potential function, but nevertheless have pure Nash equilibria. The notation in this section follows the overview article on congestion models by Voorneveld et al. (2000).

Quint and Shubik (1994) consider finite games $G=\left\langle N,\left(A_{i}\right)_{i \in N},\left(u_{i}\right)_{i \in N}\right\rangle$ satisfying the following three properties:

(QS1) There is a nonempty, finite set $F$ of facilities such that $A_{i} \subseteq F$ for all $i \in N$. 
By (QS1), an action of a player is to choose a facility from a collection $F$, possibly subject to feasibility constraints: players may not have access to all elements of $F$.

Let $a \in A, f \in F$. Let $n_{f}(a):=\left|\left\{i \in N: a_{i}=f\right\}\right|$ be the number of players choosing facility $f$ in action profile $a$.

(QS2) For each player $i \in N$ and all pure strategy profiles $a, b \in A$ with $a_{i}=b_{i}=f$ : if $n_{f}(a)=n_{f}(b)$, then $u_{i}(a)=u_{i}(b)$.

By (QS2), the utility of player $i$ depends only on the number of users of his chosen facility.

(QS3) For each player $i \in N$, each pure strategy profile $a \in A$, each player $j \in N \backslash\{i\}$ with $a_{j}=a_{i}$, and each alternative action choice $b_{j} \in A_{j} \backslash\left\{a_{j}\right\}$ of this player: $u_{i}\left(a_{j}, a_{-j}\right) \leq u_{i}\left(b_{j}, a_{-j}\right)$.

Property (QS3) models the congestion: a player is not harmed if an other user of the same facility switches to a different one. The benefit to each player from choosing a facility is weakly decreasing in the total number of users.

Proposition 5.4 Let $\Gamma^{Q S}$ be the set of Quint-Shubik congestion games, i.e., the set of games satisfying (QS1) to (QS3). For each game $G \in \Gamma^{\mathrm{QS}}$, the set of pure Nash equilibria and the collection of minimal prep sets coincide.

Proof Quint and Shubik (1994, Thm. 3) prove that $\Gamma^{\mathrm{QS}}$ has the pure Nash property. Property (QS1) allows us to restrict the set of facilities from which players make their choices: $\Gamma^{\mathrm{QS}}$ is closed w.r.t. subgames. The result follows from Proposition 3.1.

Remarks (i) The Quint-Shubik congestion games contain numerous minority games, including all our examples with payoffs as defined in (6), (7), and (9) with $g$ as in (8). Conclude that also here, there is a relevant subclass in which minimal curb sets have no cutting power.

(ii) Milchtaich (1996) allows no restrictions on access to facilities: he assumes (QS2), (QS3), and $A_{i}=F$ instead of the weaker assumption (QS1). Hence, his class of games is not closed w.r.t. subgames or minimal prep sets. Since they are special cases of the Quint-Shubik congestion games, we can nevertheless conclude that minimal prep sets and pure Nash equilibria coincide.

Acknowledgements Most of this research was carried out while Olivier Tercieux was at Tilburg University. Mark Voorneveld thanks the Netherlands Organization for Scientific Research (NWO) and the Wallander/Hedelius Foundation for financial support during parts of the research. Olivier Tercieux gratefully acknowledges financial support from the French Ministry of Research (Action Concertee Incitative). We thank Henk Norde, Willemien Kets, and two anonymous referees for valuable comments.

Open Access This article is distributed under the terms of the Creative Commons Attribution Noncommercial License which permits any noncommercial use, distribution, and reproduction in any medium, provided the original author(s) and source are credited.

\section{References}

Arthur WB (1994) Inductive reasoning and bounded rationality. Am Econ Rev 84:406-411

Basu K, Weibull JW (1991) Strategy subsets closed under rational behavior. Econ Lett 36:141-146 
Birkhoff G (1967) Lattice theory. American Mathematical Society, vol 25. Colloquium Publications

Challet D, Marsili M, Zhang Y-C (2004) Minority games: interacting agents in financial markets. Oxford University Press, New York

Challet D, Zhang Y-C (1997) Emergence of cooperation and organization in an evolutionary game. Physica A 246:407-418

Coolen ACC (2005) The mathematical theory of minority games: statistical mechanics of interacting agents. Oxford University Press, New York

Dubey P, Haimanko O, Zapechelnyuk A (2006) Strategic complements and substitutes, and potential games. Games Econ Behav 54:77-94

Hurkens S (1995) Learning by forgetful players. Games Econ Behav 11:304-329

Kalai E, Samet D (1984) Persistent equilibria in strategic games. Int J Game Theory 13:129-144

Kets W, Voorneveld M (2008) Learning to be prepared. Int J Game Theory 37:333-352

Kosfeld M, Droste E, Voorneveld M (2002) A myopic adjustment process leading to best-reply matching. Games Econ Behav 40:270-298

Koster M, Reijnierse H, Voorneveld M (2003) Voluntary contribution to multiple public projects. J Public Econ Theory 5:25-50

Luce RD, Raiffa H (1957) Games and decisions. Wiley, New York

Milchtaich I (1996) Congestion games with player-specific payoff functions. Games Econ Behav 13:111-124

Milgrom P, Roberts J (1990) Rationalizability, learning, and equilibrium in games with strategic complementarities. Econometrica 58:1255-1277

Monderer D, Shapley LS (1996) Potential games. Games Econ Behav 14:124-143

Moro E (2003) The minority game: an introductory guide. In: Korutcheva A, Cuerno R (eds) Advances in condensed matter and statistical physics. Nova Science Publishers, New York

Morris S, Ui T (2004) Best response equivalence. Games Econ Behav 49:260-287

Osborne MJ, Rubinstein A (1994) A course in game theory. MIT Press, Cambridge

Quint T, Shubik M (1994) A model of migration. Cowles Foundation Discussion Papers, No. 1088. Yale University, New Haven

Rosenthal RW (1973) A class of games possessing pure-strategy Nash equilibria. Int J Game Theory 2:65-67

Shapley LS (1964) Some topics in two-person games. In: Dresher M, Shapley LS, Tucker AW (eds) Advances in game theory. Ann. of Math. Stud., vol 52. Princeton University Press, Princeton, pp 1-28

Slade ME (1994) What does an oligopoly maximize? J Ind Econ 57:45-62

Slikker M (2001) Coalition formation and potential games. Game Econ Behav 37:436-448

Tercieux O (2006) p-Best response set. J Econ Theory 131:45-70

Topkis DM (1978) Minimizing a submodular function on a lattice. Oper Res 26:305-321

Topkis DM (1998) Supermodularity and complementarity. Princeton University Press, Princeton

Voorneveld M (1997) Equilibria and approximate equilibria in infinite potential games. Econ Lett 56:163-169

Voorneveld M (2000) Best-response potential games. Econ Lett 66:289-295

Voorneveld M (2004) Preparation. Games Econ Behav 48:403-414

Voorneveld M (2005) Persistent retracts and preparation. Games Econ Behav 51:228-232

Voorneveld M, Borm P, Megen Fvan , Tijs S, Facchini G (2000) Congestion games and potentials reconsidered. Int Game Theory Rev 1:283-299

Voorneveld M, Kets W, Norde H (2005) An axiomatization of minimal curb sets. Int J Game Theory 33:479-490

Young HP (1993) The evolution of conventions. Econometrica 61:57-84

Young HP (1998) Individual strategy and social structure. Princeton University Press, Princeton

Zhou L (1994) The set of Nash equilibria of a supermodular game is a complete lattice. Games Econ Behav 7:295-300 